\title{
Ionic liquid modified graphene for supercapacitors with high rate capability
}

\author{
Qingguo Shao, ${ }^{\mathrm{ab}} \mathrm{Jie}$ Tang, ${ }^{\mathrm{ab}}$ Yuexian Lin, ${ }^{\mathrm{ab}} \mathrm{Jing} \mathrm{Li},{ }^{\mathrm{ab}}$ Faxiang Qin, ${ }^{\mathrm{a}}$ Kun Zhang, ${ }^{\mathrm{a}}$ \\ Jinshi Yuan, ${ }^{\text {a }}$ and Lu-Chang Qin ${ }^{\text {cd }}$ \\ ${ }^{a}$ National Institute for Materials Science, 1-2-1 Sengen, Tsukuba 305-0047, Japan. E-mail: \\ tang.jie@nims.go.jp \\ ${ }^{b}$ Doctoral Program in Materials Science and Engineering, University of Tsukuba, 1-1-1 Tennodai, \\ Tsukuba 305-8577, Japan \\ ${ }^{c}$ Ningbo Institute of Materials Technology and Engineering, Chinese Academy of Sciences, Ningbo \\ 315201, China \\ ${ }^{d}$ Department of Physics and Astronomy, University of North Carolina at Chapel Hill, NC \\ 27599-3255,USA.E-mail:lcqin@unc.edu
}

\begin{abstract}
:
Ionic liquids (ILs) with large electrochemical windows up to $4 \mathrm{~V}$ have been employed as the electrolyte to boost the energy density of graphene-based supercapacitors. However, due to the larger molecular size, lower conductivity, and higher viscosity of the IL electrolyte, graphene-based supercapacitors in IL electrolyte usually exhibit rate capability. To make graphene-based electrodes more compatible with the IL electrolyte, we functionalized chemically reduced graphene oxide with the same IL which is also used as the electrolyte. Electrochemical test results show that the


relaxation time and charge transfer resistance at electrode-electrolyte interface for IL modified electrode is one third and one fourth of that for the pristine graphene respectively, indicating the improved compatibility between the IL modified electrode and the electrolyte. Furthermore, the capacitance retention of the IL modified from current density of 0.5 to $20 \mathrm{~A} \mathrm{~g}^{-1}$ is $85 \%$, which is much higher than that of the pristine electrode $(53 \%)$.

\section{Key words:}

Graphene; Ionic liquid; Supercapacitor; Rate capability

*Corresponding author. Jie Tang, Email: tang.jie@ nims.go.jp;

Tel: +81-29-859-2728; Fax: +81-29-859-2701 


\section{Introduction}

Electric double-layer capacitors (EDLC), also often called supercapacitors, as a kind of energy storage device, have received great attention worldwide because of unique characteristics including high power density, long cyclic lifetime, and low maintenance cost. These features make supercapacitors a potential versatile power supply source for portable electronic devices, hybrid electric vehicles, solar energy systems, and memory backups, especially where peak power is demanded. ${ }^{1-10}$ In the recent years, graphene, a monolayer carbon material with large theoretical specific surface area of $2600 \mathrm{~m}^{2} \mathrm{~g}^{-1}$ and high thermal and electric conductivity, has been considered as an ideal electrode material for supercapacitors. ${ }^{11-20}$ For graphene-based supercapacitors, one challenging and really important issue is to increase their energy density. The energy density $(E)$ of a supercapacitor is related to the specific (C) and operating voltage $(V)$ by $E=C V^{2} / 2$. Therefore, raising the operating voltage crucial to the increase of the energy density of supercapacitors. In general, the voltage of supercapacitors is limited by the electrochemical window of the electrolyte. Compared with aqueous electrolytes, ionic liquids (ILs) with an original electrochemical window up to $4 \mathrm{~V}$ have been employed as the electrolytes to boost the energy density of graphene-based supercapacitors. ${ }^{21-23}$ For example, we have recently reported that graphene-based electrodes could exhibit high energy densities of 80-150 $\mathrm{Wh} \mathrm{kg}^{-1}$ in IL electrolyte. ${ }^{24-28}$

However, apart from the relatively high cost, there still exists another obstacle for the application of IL as the electrolyte for supercapacitors. Compared with aqueous 
electrolytes, ILs usually have larger molecules, lower conductivity, and higher viscosity, graphene supercapacitors often exhibit lower rate capability. This is an important issue, because if the supercapacitor could not be charged and discharged quickly, the projected high energy density due to the IL electrolyte would not be materialized. To address this issue, organic solvents and/or single-walled carbon nanotubes have been added into the IL electrolyte to reduce the viscosity and increase the ionic conductivity. ${ }^{29,30}$ However, these efforts are all focused on improving the properties of the IL electrolyte and there is a lack of attention on modifying the surface of the electrodes to make them more compatible with the IL electrolyte. Recently, there have been a few published reports on IL-graphene composites as biosensors and supercapacitor electrodes showing improved electrochemical performance. $^{31-33}$

In this study a commonly used IL electrolyte, 1-ethyl-3-methylimidazolium tetrafluoroborate $\left(\mathrm{EMIMBF}_{4}\right)$, has been used directly as a functionalizer to modify the graphene surface and it has also been used as the electrolyte for assembling the supercapacitor. Our aim is to improve the compatibility between the IL modified graphene surface and the same IL electrolyte. This idea is based on the fact that the electric double-layer capacitance comes from the ion adsorption on the surface of the electrodes and therefore tailoring the surface chemistry of the electrodes would be an effective approach to improve their capacitive behavior. Actual electrochemical measurements showed that the relaxation time and charge transfer resistance at the electrode-electrolyte interface for the IL modified electrode is one third and one 
fourth of that for the pristine electrode, respectively, indicating the improved performance between the IL modified electrode and the electrolyte. More importantly, different from previous methods focused on improving the IL electrolyte, our approach shed some light on new strategies to improve the rate capability of the IL electrolyte supercapacitors.

\section{Experimental}

\subsection{Preparation of Graphene Oxide}

Graphene oxide (GO) was prepared from natural graphite powders according to a modified Hummers' method. ${ }^{34}$ In brief, graphite powders were mixed with sulfuric acid and sodium nitrate in an ice-bath. After stirring, potassium permanganate was slowly added so that the temperature would not exceed $20^{\circ} \mathrm{C}$. The reaction system was transferred to a $35^{\circ} \mathrm{C}$ water bath for 1 hour, forming a thick paste. After that, deionized water was added gradually into the mixture and stirred for another hour, followed by an addition of water. After 30 minutes, $\mathrm{H}_{2} \mathrm{O}_{2}$ was slowly added and the colour would change from dark brown to yellow. The suspension was then centrifuged and washed with $\mathrm{HCl}$ solution and deionized water for six times to remove the metal ions and residual acid. The obtained product was dried under vacuum to obtain GO powders. Finally, the GO powders were dispersed in water by sonication for 2 hours to make a homogeneous aqueous dispersion of GO with a concentration of $1 \mathrm{mg} \mathrm{mL}^{-1}$.

\subsection{Preparation of Reduce Graphene Oxide}


Reduced graphene oxide (RGO) was prepared by chemical reduction of the prepared GO. The aqueous dispersion of GO $\left(1 \mathrm{mg} \mathrm{mL}^{-1}\right)$ was stirred in a flask. Then, it was heated to $98{ }^{\circ} \mathrm{C}$ and hydrazine solution (35 wt $\%$ ) was added. The mixture was kept at $98{ }^{\circ} \mathrm{C}$ for 2 hours under reflux. Finally, the product was washed with water and dried in an oven at $60{ }^{\circ} \mathrm{C}$ to obtain reduced graphene oxide (RGO).

\subsection{Preparation of Ionic Liquid Modified Reduced Graphene Oxide}

Ionic liquid modified reduced graphene oxide (IL-RGO) was prepared by chemical reduction of $\mathrm{GO}$ in the ionic liquid. $100 \mathrm{~mL}$ aqueous $\mathrm{GO}$ dispersion $(1 \mathrm{mg}$ $\left.\mathrm{mL}^{-1}\right)$ was first stirred in a flask with different amount $(1 \mathrm{~mL}, 2 \mathrm{~mL}$, and $5 \mathrm{~mL})$ of ionic liquid 1-ethyl-3-methylimidazolium tetrafluoroborate $\left(\mathrm{EMIMBF}_{4}\right)$ added into the suspension. After stirring for 30 minutes, it was heated to $98{ }^{\circ} \mathrm{C}$ and hydrazine solution $(35 \mathrm{wt} \%)$ was added. The mixture was kept at $98{ }^{\circ} \mathrm{C}$ for 2 hours under reflux. Finally, the product was washed with water and dried in an oven at $60{ }^{\circ} \mathrm{C}$. The obtained IL-RGO samples were denoted as IL-RGO-1, IL-ROG-2, IL-RGO-5 according to the amount of IL added in the mixture.

\subsection{Structural Characterization}

The morphology and structure of material samples were examined with scanning electron microscope (SEM) using JSM-6500 field-emission SEM with acceleration voltage of $15 \mathrm{KV}$. Transmission electron microscopy (TEM) was carried out using a JEM-2100 electron microscope. Atomic force microscopy (AFM) was performed using an Agilent AFM-550. Fourier transformation infrared (FT-IR) spectra were recorded using a Nicolet 6700 spectrometer. The specific surface area (SSA) and pore 
size distribution were measured by the BET method using an Autosorb-iQ analyzer. The SSA value was calculated by the BET method based on the adsorption data in the relative pressure $\left(\mathrm{P} / \mathrm{P}_{0}\right)$ range of 0.05 to 0.3 .

\subsection{Electrochemical Characterization}

Two-electrode symmetrical supercapacitors were assembled and used for evaluating the electrochemical performance of the as-obtained electrode samples. In preparation of the supercapacitor electrodes, $90 \mathrm{wt} \%$ graphene and $10 \mathrm{wt} \%$ PTFE were dispersed in ethanol by sonication and then the suspension was filtered onto a porous filter membrane by vacuum filtration to obtain filtered films. After vacuum drying for 24 hours, all electrodes were prepared by cutting the filtered films into circular disks with a diameter of $15 \mathrm{~mm}$ and weight of about $1 \mathrm{mg}$ for each electrode. The two-electrode cell was assembled in a glove box filled with Ar. A porous separator was placed between the two electrodes in a stainless cell. $\mathrm{EMIMBF}_{4}$ was used as the ionic liquid electrolyte.

Electrochemical measurements were carried out at room temperature using a multi-channel VMP-300 electrochemical workstation. The voltage range for cyclic voltammetry $(\mathrm{CV})$ measurements and galvanostatic charge-discharge tests was 0 - 3.5 V. The gravimetric specific capacitance, $C_{s}\left(\mathrm{~F} \mathrm{~g}^{-1}\right)$ was calculated according to $\mathrm{C}_{\mathrm{s}}=\quad \ldots \ldots=4 I /(\mathrm{m} d V / d t)$, where $I(\mathrm{~A})$ is the constant current, $m(\mathrm{~g})$ is the total mass of the two electrodes, and $d V / d t\left(\mathrm{~V} \mathrm{~s}^{-1}\right)$ is the slope obtained by fitting a straight line to the discharge curve between $V_{\max }$ (the voltage at the beginning of discharge) and $1 / 2 V_{\text {max. }}$ In the Ragone plot, the energy density, $E_{\text {cell }}\left(\mathrm{Wh} \mathrm{kg}^{-1}\right)$, was calculated by $E_{\text {cell }}$ 
$=C_{s} V^{2} / 8$. The power density, $P_{\text {cell }}\left(\mathrm{W} \mathrm{kg}^{-1}\right)$, was calculated by $P_{\text {cell }}=E_{\text {cell }} / t$, where $t$ is the discharge time. Electrochemical impedance spectroscopy (EIS) measurements were carried out in the frequency range of $10^{-2}-10^{5} \mathrm{~Hz}$ at open circuit potential with an $\mathrm{AC}$ amplitude of $5 \mathrm{mV}$. Based on an $\mathrm{RC}$ model, the capacitance was also calculated from the frequency response analysis, using $C=-1 /\left(2 \pi f Z^{\prime \prime}\right)$, to show the trend of changes in capacitance with frequency, where $f$ is frequency $(\mathrm{Hz})$ and $Z$ " is the imaginary part of the impedance.

\section{Results and Discussion}

The morphology and structure of RGO and IL-RGO were all examined with SEM. Fig. 1a and $\mathrm{b}$ are the SEM images of RGO in which a stacked planar structure due to the $\pi-\pi$ interactions and the van der Waals forces between graphene layers. This compact structure would apparently reduce the specific surface area. Fig. 1c-h shows the SEM images of IL-RGO prepared with 1, 2, and $5 \mathrm{~mL}$ of IL, respectively. Compared with RGO, all the three IL-RGO samples showed a much more porous structure and the porousness increases with increasing amount of IL. In addition, the IL-RGO samples also showed a bended structure (as displayed in the enlarged SEM images Fig. 1d-h) and these bended sheets formed a three-dimensional (3D) structure, which is different from the stacked planar nature of RGO. In this case, this loosely packed 3D structure would be favorable for electrolyte infiltration to provide more exposed surface area for ion adsorption in order to improve the supercapacitor performance. It should also be noted that the morphological changes are a commonly 
observed phenomenon in the IL mediated reactions, especially in the synthesis of certain kind of inorganic compounds with unique structures. ${ }^{35,36}$ Here, in this system, considering the good dispersibility of graphene in $\mathrm{IL},{ }^{37}$ the IL is suggested to have served as a surfactant and a soft template to make the freshly reduced graphene sheets well separated and bended.

Transmission electron microscopy (TEM) was employed to further characterize the structure of the as-prepared graphene material samples. Fig. 2a shows the TEM image of RGO and Fig. 2b-d show the TEM images of IL-RGO with different degree of modification. It can be seen that the graphene sheets are thick stacks in the pristine RGO sample. On the other hand, in the IL-RGO samples (Fig. 2b-d), corrugated features are typical and the IL-RGO samples are more separated with increasing degree of IL modification. These TEM results are in good agreement with the SEM observations shown in Fig. 1. Furthermore, as revealed in the TEM images, the stacks in the IL-RGO samples are thinner than that in the RGO sample. This observation is further confirmed using AFM measurement. The RGO stack is typically $6.95 \mathrm{~nm}$ in thickness and the IL-RGO stack is about $1.30 \mathrm{~nm}$ (see Supplementary Information, Fig. S1).

The IL induced surface modification was verified by FT-IR analysis (Fig. 3). Additional adsorption bands due to IL $\left(\mathrm{EMIMBF}_{4}\right)$ at 3160 and 3090, 1572, and 1065 $\mathrm{cm}^{-1}$, corresponding to the stretching vibrations of unsaturated $\mathrm{CH}_{\mathrm{x}}$ bonds, $\mathrm{C}-\mathrm{N}$ bonds from the imidazolium ring, and the $\mathrm{B}-\mathrm{F}$ bonds of $\left[\mathrm{BF}_{4}\right]^{-}$anions, respectively, were observed in the spectra of IL-RGO, implying successful modification of the reduced 
graphene with the $\mathrm{IL} .^{38}$

The specific surface area (SSA) and the pore structure of RGO and IL-RGO were studied by nitrogen isothermal adsorption (Fig. 4). The adsorption isotherm of RGO reveals a characteristic of type IV, which is typical for the adsorption of mesopores. However, IL-RGO exhibits an isotherm combining type III and type IV, indicating that macropores also exists in IL-RGO in addition to large mesopores. The existence of large mesopores is further verified in the pore size distribution (Fig. 4b), which confirms the pores in the range of $20-150 \mathrm{~nm}$. The presence of macropores is consistent with the results of SEM and TEM observations that showed porous structures. It should also be noted that these macropores induced by IL mediated reduction would help to improve the rate capability of the IL-RGO electrode because the electrolyte ion diffusion to macropores would be much easier than to mesopores. Besides, the peak pore diameter of IL-RGO in the pore size distribution plot is a little larger than that of RGO (shown in inset of Fig. 4b), which further confirmed that the graphene sheets are more separated due to the "surfactant" IL. Benefited from the well-separated structure, a larger SSA of $433 \mathrm{~m}^{2} \mathrm{~g}^{-1}$ for IL-RGO was obtained compared with RGO $\left(324 \mathrm{~m}^{2} \mathrm{~g}^{-1}\right)$.

The supercapacitor performance with RGO and IL-RGO electrodes was investigated with a two-electrode symmetrical configuration using the same ionic liquid $\left(\mathrm{EMIMBF}_{4}\right)$ as the electrolyte. Fig. $5 \mathrm{a}$ and $5 \mathrm{~b}$ show the comparative cyclic voltammetry (CV) curves of RGO and IL-RGO (with $2 \mathrm{~mL}$ IL) electrodes at low and high scanning rate, respectively (comparison of electrochemical performance with 

scanning rate of $50 \mathrm{mV} \mathrm{s}^{-1}$, both $\mathrm{CV}$ profiles exhibit rectangular shapes, indicating their idealistic electric double-layer capacitance. However, when the scanning rate is increased to ten times, rectangular shaped CV curves can only be observed for the IL-RGO electrode, demonstrating its superior rate capability. This trend is more obvious in the galvanostatic charge-discharge results, which shows that the discharge time of IL-RGO electrode is significantly larger than that of RGO at high current density of $20 \mathrm{~A} \mathrm{~g}^{-1}$ (Fig. 5d). The dependence of gravimetric specific capacitance on current density is presented in Fig. 5e. At a low current density of $0.5 \mathrm{~A} \mathrm{~g}^{-1}$, the IL-RGO electrode exhibits a slightly higher capacitance of $135 \mathrm{~F} \mathrm{~g}^{-1}$ than RGO (129 F $\mathrm{g}^{-1}$ ), which may be due to the larger SSA of IL-RGO. When the current density increases further by forty times, the retained capacitance of the RGO electrode is only $68 \mathrm{~F} \mathrm{~g}^{-1}$, corresponding to a low retention of $53 \%$, revealing its poor rate capability owing to the low conductivity and high viscosity of the IL electrolyte. However, after IL modification, a high specific capacitance of $114 \mathrm{~F} \mathrm{~g} \mathrm{~g}^{-1}$, corresponding to a large retention of $85 \%$, was observed for the IL-RGO electrode under the same condition. This high retention is much better than the untreated RGO electrode and even comparable to some reported RGO electrode in aqueous electrolytes, ${ }^{39-41}$ evidently revealing the significant importance of IL modification to the improvement of rate capability for graphene supercapacitors in IL electrolyte. Furthermore, the Ragone plots shown in Fig. 5f further demonstrated the improved rate performance of the IL-RGO electrode. Even at a high power density of $18 \mathrm{~kW} \mathrm{~kg}^{-1}$, the IL-RGO electrode 
still delivered a high energy density of $49 \mathrm{Wh} \mathrm{kg}^{-1}$, which is much larger than that of RGO (29 $\left.\mathrm{Wh} \mathrm{kg}^{-1}\right)$. Additionally, the IL-RGO electrode exhibits excellent cycling stability (Fig. 5g). It can retain over $92 \%$ of capacitance after 2000 cycles even under a high current density of $10 \mathrm{~A} \mathrm{~g}^{-1}$, which is much improved over the RGO electrode $(82 \%)$

To understand the reasons for the improved rate capability after IL modification, electrochemical impedance spectroscopy (EIS) was applied to examine the interfacial properties of the RGO and IL-RGO electrodes with the IL electrolyte. Fig. 6a displays the Nyquist plots of RGO and IL-RGO based supercapacitors. The equivalent series resistance (ESR) from the intercept on the $\mathrm{x}$-axis for IL-RGO is lower than RGO, which can be ascribed to better contact with the current collector due to its curved structure with more exposed edges of graphene that provide numerous contact points for charge transport. The radius of the semicircle in the Nyquist plot indicates the charge transfer resistance $\left(R_{c t}\right)$, which is closely related to the transport of electrolyte ions at the electrode/electrolyte interface. ${ }^{42}$ After IL modification, the value of $R_{c t}$ for IL-RGO is $0.86 \Omega$, which is only a quarter of that for RGO $(3.5 \Omega)$, demonstrating the improved compatibility between the IL modified electrode and the IL electrolyte. The largely reduced resistance would facilitate fast ion adsorption/desorption, thus improving the rate capability of the supercapacitor. Moreover, Fig. $6 \mathrm{~b}$ shows the frequency response of the RGO and IL-RGO electrodes in the range from $10 \mathrm{mHz}$ to $100 \mathrm{kHz}$. The operating frequency $f_{0.5}$ (the frequency at which the capacitance is $50 \%$ of its maximum value) $)^{42}$ of IL-RGO and RGO is $18.60 \mathrm{~Hz}$ and $5.75 \mathrm{~Hz}$, respectively, 
which corresponds to a relaxation time constant $\tau_{0}\left(=1 / f_{0.5}\right)$ of $53.6 \mathrm{~ms}$ for IL-RGO, about one-third of that of RGO (173.9 ms). This rapid frequency response of IL-RGO further confirms the significantly improved compatibility between the IL modified electrode and the IL electrolyte. Three main reasons are suggested to account for the improved compatibility between the IL modified graphene electrode and the IL electrolyte. Firstly, the porous and curved structure, induced by the IL "surfactant", could effectively reduce the restacking of graphene, thus providing a more electrolyte-accessible structure that can be quickly wetted by the electrolyte. Secondly, the presence of IL molecules on the outermost surface of graphene sheets would reduce the adsorption free energy when encountering the same kind of IL electrolyte to promote ion adsorption. Thirdly, the IL molecules that inserted between the graphene sheets would open up additional ion diffusion paths to accelerate ion transport.

\section{Conclusions}

Surface modification of RGO electrode by IL is an effective way to improve the rate capability of supercapacitors using the same IL electrolyte. The modification with IL can significantly improve the compatibility between the electrode and the IL electrolyte. Electrochemical tests reveal that the IL modified electrode can exhibit much higher capacitance retention under high current density. More importantly, different from previous effort focused on improving IL electrolyte, this study potentially opens up a new, simple, and effective route to improve the rate capability of graphene based supercapacitors in IL electrolyte. 


\section{Acknowledgements}

This work was supported by JST ALCA program, JSPS Grants-in-aid for Scientific Research (No. 22310074), the Nanotechnology Network Project of the Ministry of MEXT, Japan and Provincial Natural Science Foundation (No. LY14E020010) of Zhejiang, China.

\section{References}

1. G. Wang, L. Zhang and J. Zhang, Chemical Society Reviews, 2012, 41, 797-828.

2. G. Yu, X. Xie, L. Pan, Z. Bao and Y. Cui, Nano Energy, 2013, 2, 213-234.

3. P. Simon and Y. Gogotsi, Nature Materials, 2008, 7, 845-854.

4. D. Zhang, X. Zhang, X. Sun, H. Zhang, C. Wang and Y. Ma, Electrochimica Acta, 2013, 109, 874-880.

5. Q. Cheng, J. Tang, J. Ma, H. Zhang, N. Shinya and L.-C. Qin, The Journal of Physical Chemistry C, 2011, 115, 23584-23590.

6. L. Borchardt, M. Oschatz and S. Kaskel, Materials Horizons, 2014, 1, 157.

7. Y. Huang, J. Liang and Y. Chen, Small, 2012, 8, 1805-1834.

8. C. Marichy, M. Bechelany and N. Pinna, Advanced Materials, 2012, 24, 1017-1032.

9. A. L. Mohana Reddy, S. R. Gowda, M. M. Shaijumon and P. M. Ajayan, Advanced Materials, 2012, 24, 5045-5064. 
10. W. Wei, X. Cui, W. Chen and D. G. Ivey, Chemical Society Reviews, 2011, 40, 1697-1721.

11. M. D. Stoller, S. Park, Y. Zhu, J. An and R. S. Ruoff, Nano Letters, 2008, 8, 3498-3502.

12. Y. Zhu, S. Murali, M. D. Stoller, K. J. Ganesh, W. Cai, P. J. Ferreira, A. Pirkle, R. M. Wallace, K. A. Cychosz, M. Thommes, D. Su, E. A. Stach and R. S. Ruoff, Science, 2011, 332, 1537-1541.

13. W. J. Lee, U. N. Maiti, J. M. Lee, J. Lim, T. H. Han and S. O. Kim, Chemical Communications, 2014, 50, 6818-6830.

14. B. Luo, S. Liu and L. Zhi, Small, 2012, 8, 630-646.

15. Y. B. Tan and J.-M. Lee, Journal of Materials Chemistry A, 2013, 1, 14814.

16. M. Zhou, T. Tian, X. Li, X. Sun, J. Zhang, Y. Chen, P. Cui, J. Tang and L.-C. Qin, Chemical Physics Letters, 2013, 581, 64-69.

17. D. Chen, L. Tang and J. Li, Chemical Society Reviews, 2010, 39, 3157.

18. A. Ghosh and Y. H. Lee, ChemSusChem, 2012, 5, 480-499.

19. L. L. Zhang, R. Zhou and X. S. Zhao, Journal of Materials Chemistry, 2010, 20, 5983.

20. D. A. C. Brownson, D. K. Kampouris and C. E. Banks, Journal of Power Sources, 2011, 196, 4873-4885.

21. Z. Lei, Z. Liu, H. Wang, X. Sun, L. Lu and X. S. Zhao, Journal of Materials Chemistry A, 2013, 1, 2313. 
22. W.-Y. Tsai, R. Lin, S. Murali, L. Li Zhang, J. K. McDonough, R. S. Ruoff, P.-L. Taberna, Y. Gogotsi and P. Simon, Nano Energy, 2013, 2, 403-411.

23. H. Zhong, F. Xu, Z. Li, R. Fu and D. Wu, Nanoscale, 2013, 5, 4678-4682.

24. Q. Cheng, J. Tang, J. Ma, H. Zhang, N. Shinya and L. C. Qin, Physical Chemistry Chemical Physics : PCCP, 2011, 13, 17615-17624.

25. F. Zhang, J. Tang, N. Shinya and L.-C. Qin, Chemical Physics Letters, 2013, 584, 124-129.

26. Q. Shao, J. Tang, Y. Lin, F. Zhang, J. Yuan, H. Zhang, N. Shinya and L.-C. Qin, Journal of Materials Chemistry A, 2013, 1, 15423.

27. Q. Shao, J. Tang, Y. Lin, J. Li, F. Qin, J. Yuan and L.-C. Qin, Journal of Power Sources, 2015, 278, 751-759.

28. Q. Cheng, J. Tang, J. Ma, H. Zhang, N. Shinya and L.-C. Qin, Carbon, 2011, 49, 2917-2925.

29. V. Ruiz, T. Huynh, S. Sivakkumar and A. Pandolfo, RSC Advances, 2012, 2, 5591-5598.

30. C. Kong, W. Qian, C. Zheng, Y. Yu, C. Cui and F. Wei, Chemical Communications, 2013, 49, 10727.

31. C. Shan, H. Yang, D. Han, Q. Zhang, A. Ivaska, L. Niu, Biosensors \& Bioelectronics, 2010, 25, 1504-1508.

32. J. Peng, C. Hou, X. Hu, Sensors and Actuators B: Chemical, 2012, 169, 81-87.

33. T.Y. Kim, H.W. Lee, M. Stoller, D.R. Dreyer, C.W. Bielawski, R.S. Ruoff, K.S. Suh, ACS Nano, 2010, 5, 436-442. 
34. Y. Zhao, C. Hu, L. Song, L. Wang, G. Shi, L. Dai, L. Qu, Energ and Environmental Science, 2004, 7, 1913-1918.

35. B. L. Cushing, V. L. Kolesnichenko and C. J. O Connor, Chemical Reviews, 2004, 104, 3893-3946.

36. Z. Ma, J. Yu and S. Dai, Advanced Materials, 2010, 22, 261-285.

37. X. Zhou, T. Wu, K. Ding, B. Hu, M. Hou and B. Han, Chemical Communications, 2010, 46, 386-388.

38. K. Liu, J. Zhang, G. Yang, C. Wang and J.-J. Zhu, Electrochemistry Communications, 2010, 12, 402-405.

39. Y. Xu, Z. Lin, X. Huang, Y. Liu, Y. Huang and X. Duan, ACS Nano, 2013, 7, $4042-4049$.

40. Z. Lei, L. Lu and X. S. Zhao, Energy \& Environmental Science, 2012, 5, 6391.

41. F. Liu, S. Song, D. Xue and H. Zhang, Advanced Materials, 2012, 24, 1089-1094.

42. L. Zhang, F. Zhang, X. Yang, G. Long, Y. Wu, T. Zhang, K. Leng, Y. Huang, Y. Ma, A. Yu and Y. Chen, Scientific Reports, 2013, 3, 1408.

43. X. Yang, J. Zhu, L. Qiu and D. Li, Advanced Materials, 2011, 23, 2833-2838. 


\section{Figure Captions}

Figure 1. SEM images of (a,b) RGO and (c-h) IL-RGO with different degrees of modification: (c,d) with $1 \mathrm{~mL}$ IL (IL-RGO-1); (e,f) with 2mL IL (IL-RGO-2); and (g,h) with $5 \mathrm{~mL}$ IL (IL-RGO-5). IL-RGOs exhibit a porous structure with corrugated bended sheets, while RGO shows a compact and less porous structure.

Figure 2. TEM images of (a) RGO; (b) IL-RGO-1 (with 1mL IL); (c) IL-RGO-2 (2 mL IL); and (d) IL-RGO-5 (with $5 \mathrm{~mL}$ IL). The IL-RGO sheets are much thinner than the stacked pristine RGO sheets.

Figure 3. FT-IR spectra of RGO and IL-RGO. The existence of adsorption bands of IL $\left(\mathrm{EMIMBF}_{4}\right)$ in the spectra of IL-RGO confirms the IL modification to the reduced graphene.

Figure 4. (a) Nitrogen adsorption isotherms and (b) BJH desorption pore size distribution curves of RGO and IL-RGO-2. The peak pore diameter of IL-RGO in the pore size distribution plot is a little larger than that of RGO, which further confirms the more separated graphene sheets.

Figure 5. Supercapacitor performance of RGO and IL-RGO electrodes in IL electrolyte using a two-electrode cell. (a,b) CV curves obtained at slow and high scan rates; (c,d) Charge/discharge curves obtained at different current densities; (e) 
Gravimetric specific capacitance measured at various charge/discharge current densities; (f) Ragone plots; and (g) Cycling stability at a current density of $10 \mathrm{~A} \mathrm{~g}^{-1}$.

Figure 6. (a) Nyquist plots of RGO and IL-RGO electrodes. The inset shows an expanded portion at high frequency. (b) Frequency response of RGO and IL-RGO electrodes. The relaxation time and charge transfer resistance for the IL modified electrode is one third and one fourth of that for the pristine electrode, respectively, indicating improved compatibility between the IL modified electrode and the electrolyte. 


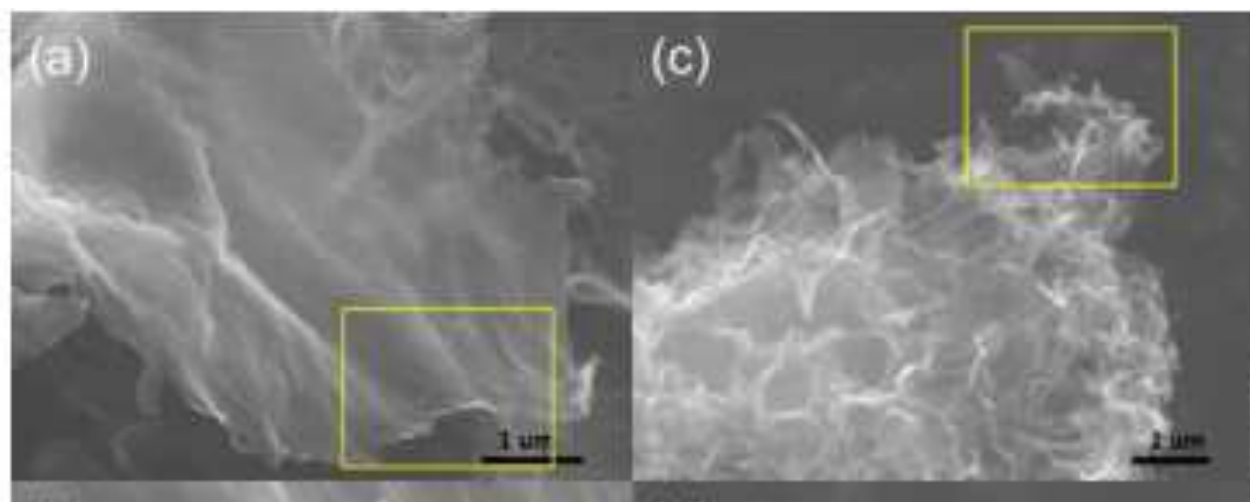

(b)

(d)

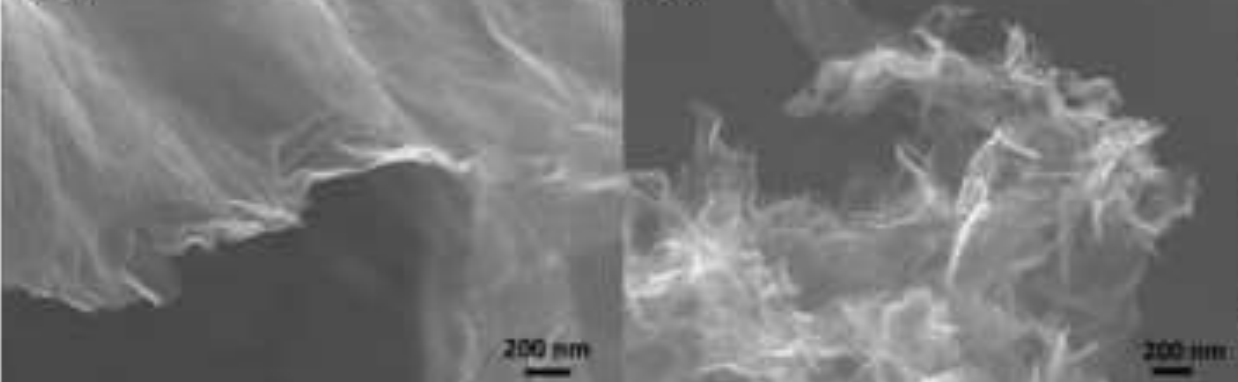

(e)
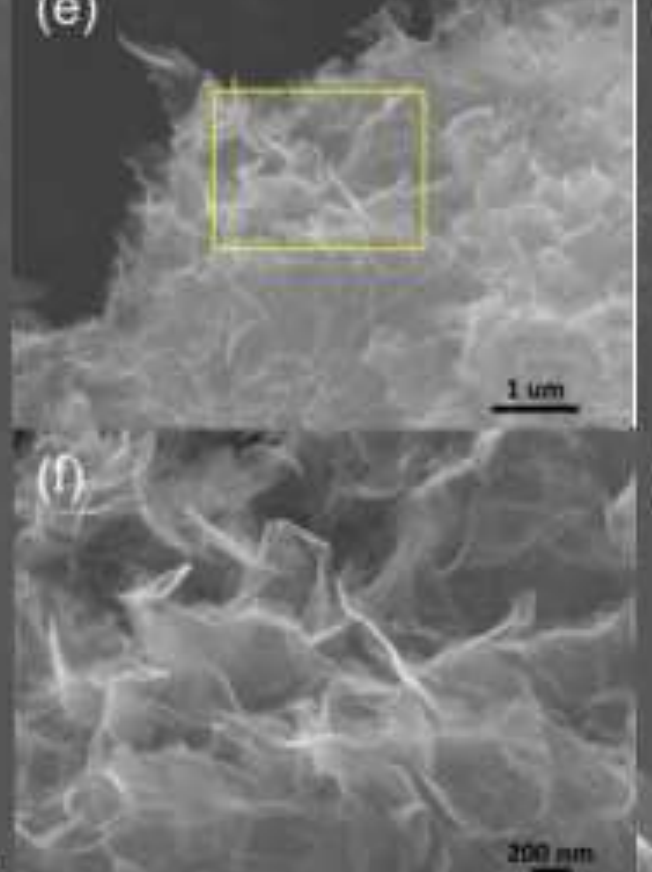

(g)

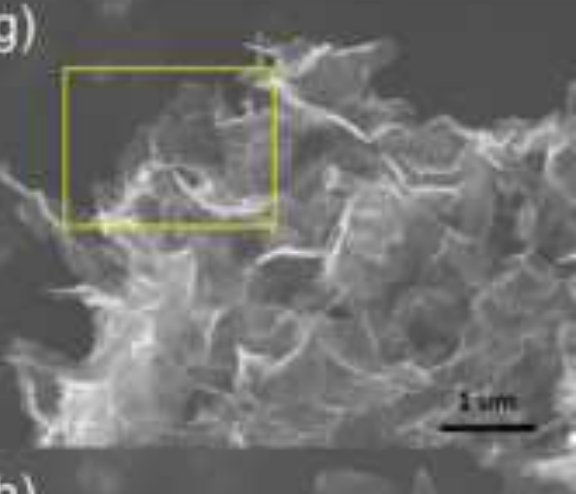

(h)

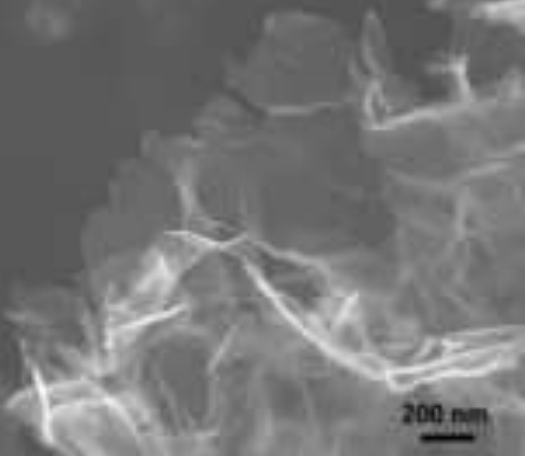



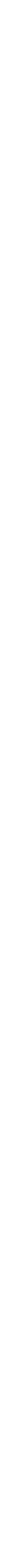


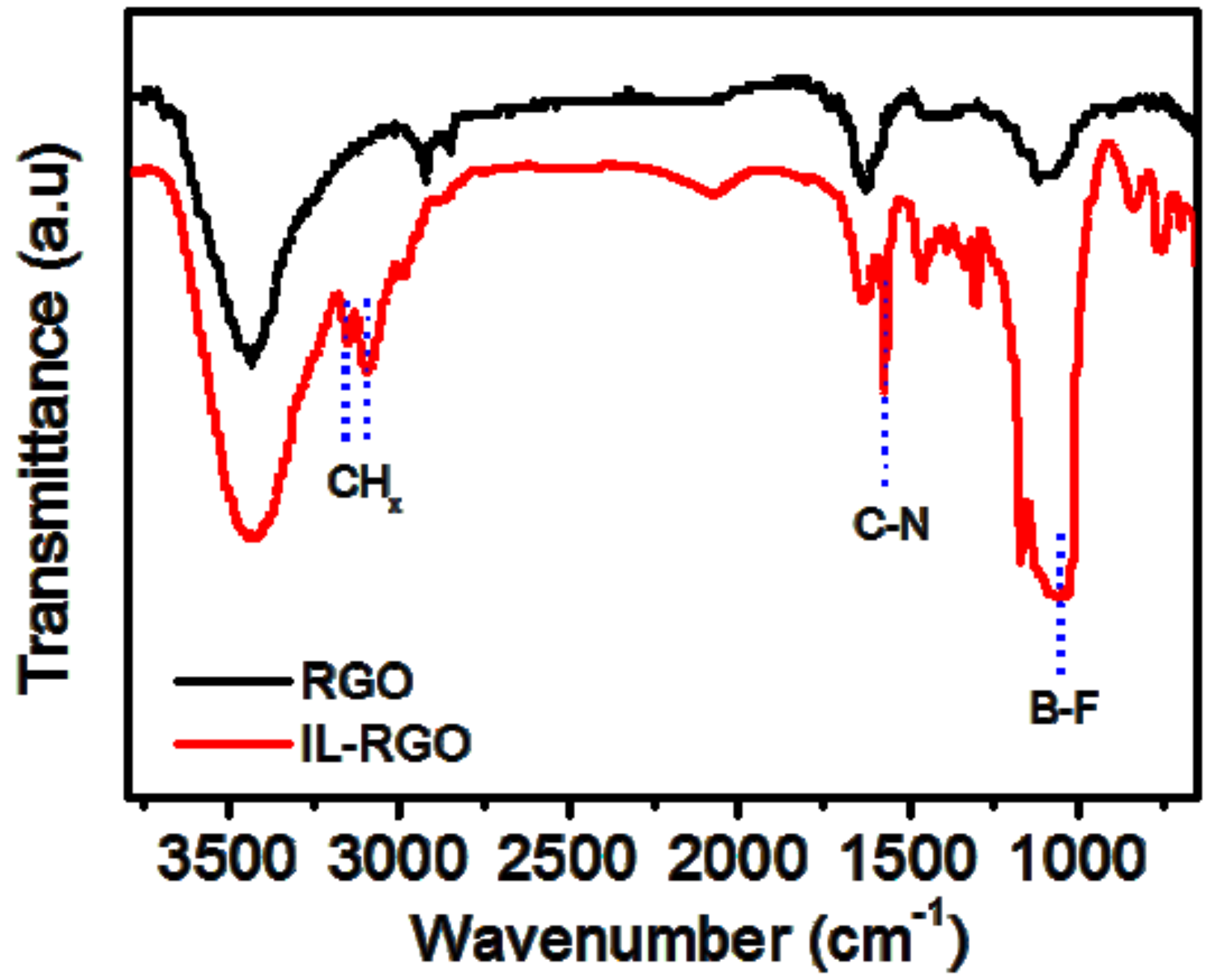


(a)

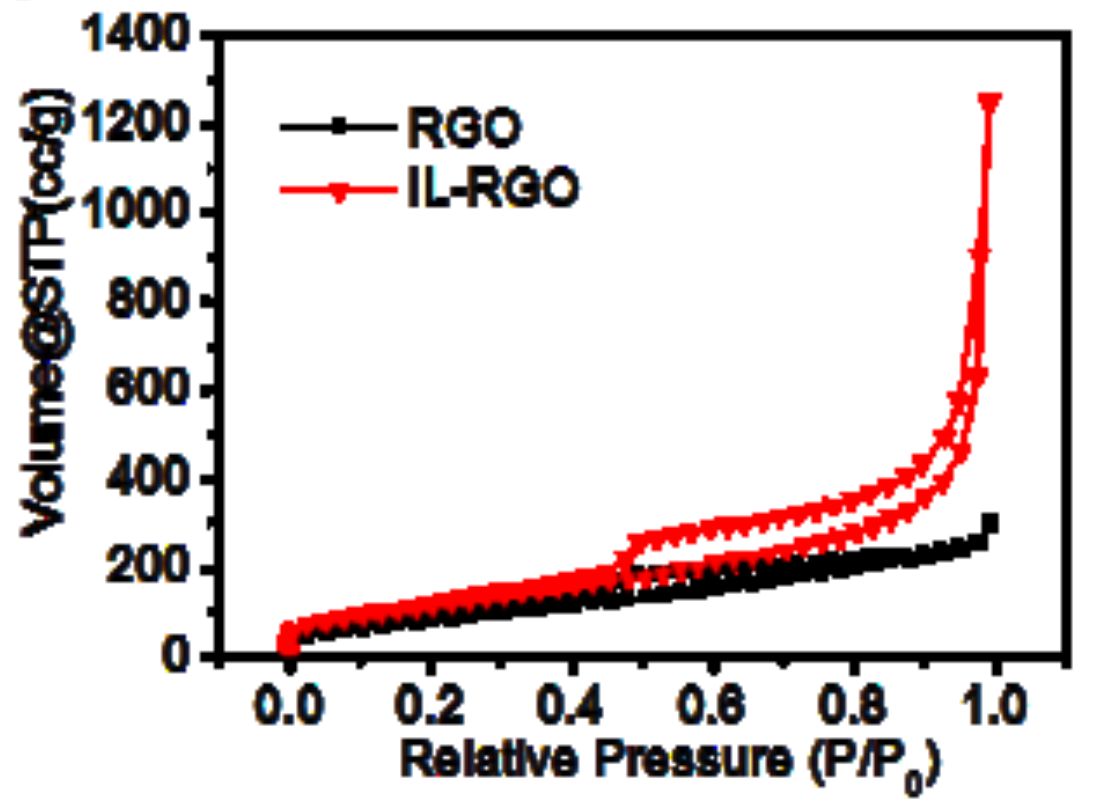

(b)

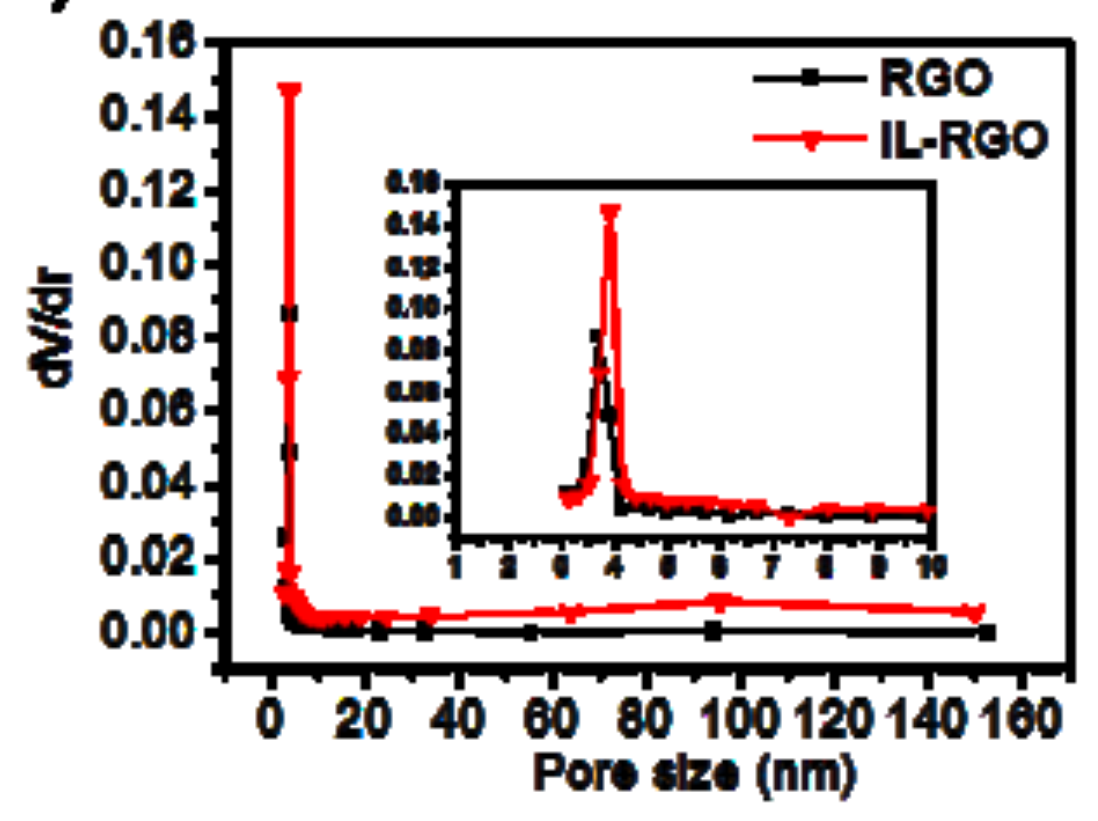


Figure 5

(a)

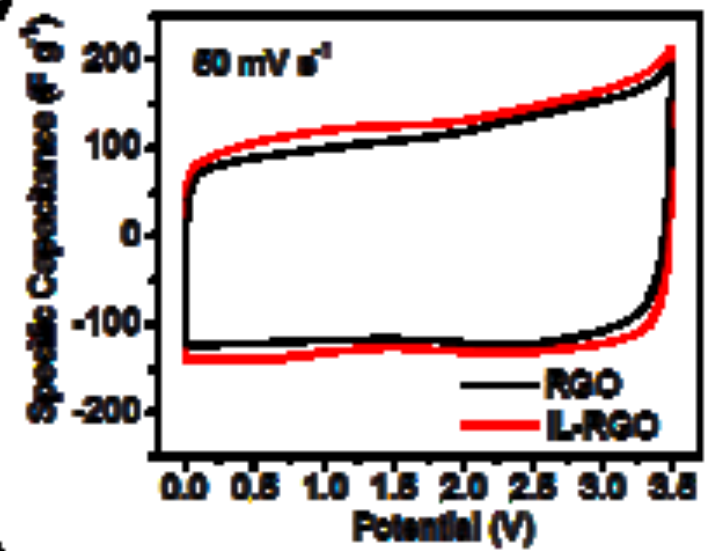

(c)

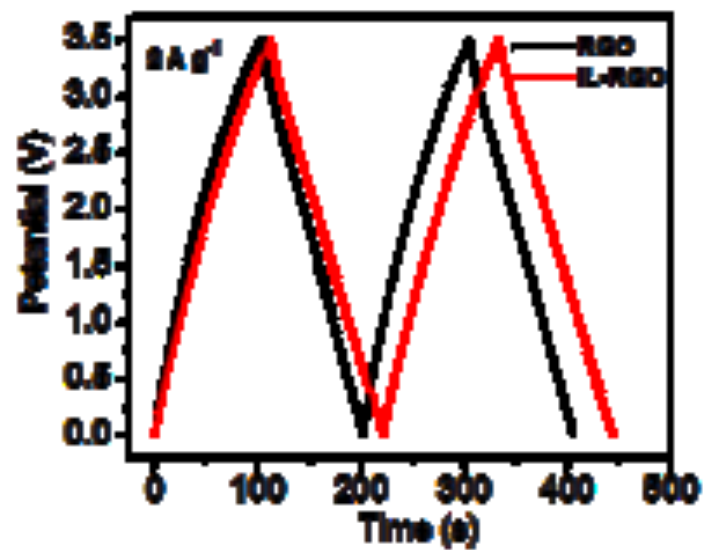

(e)

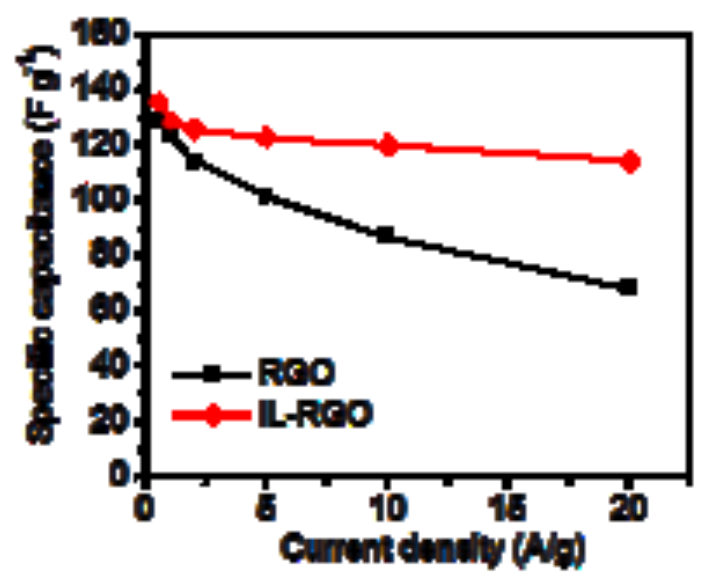

(b)

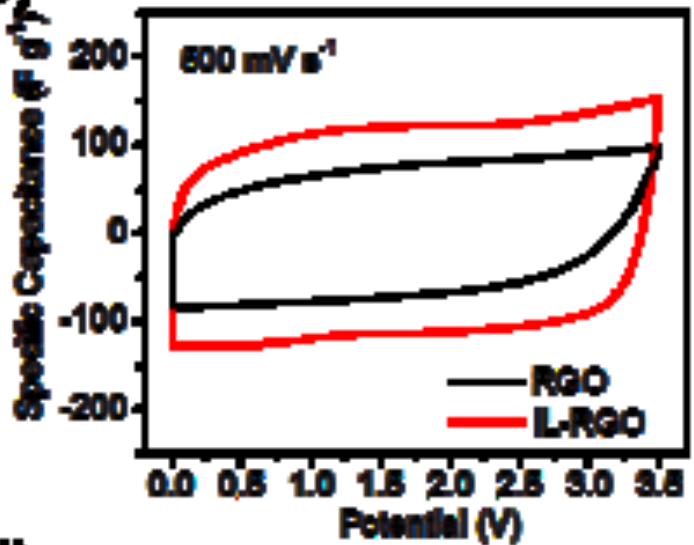

(d)

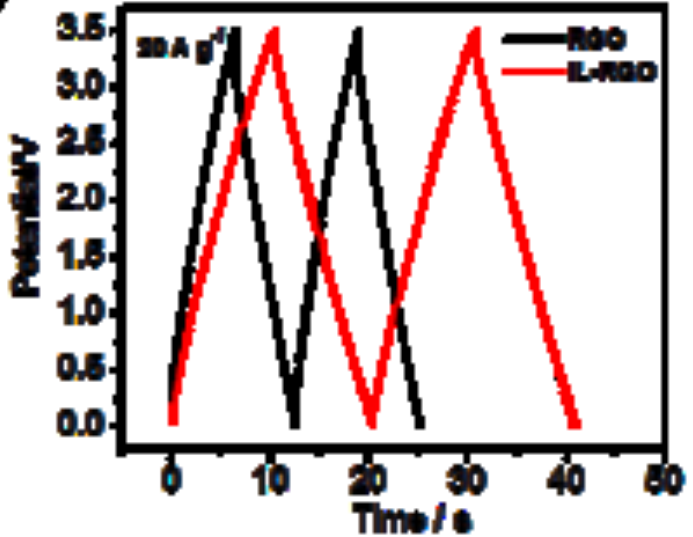

(f)

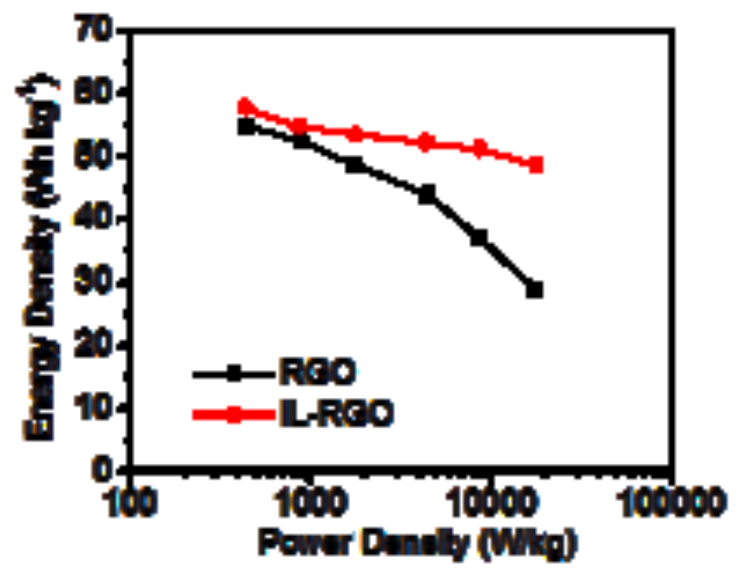

(g)

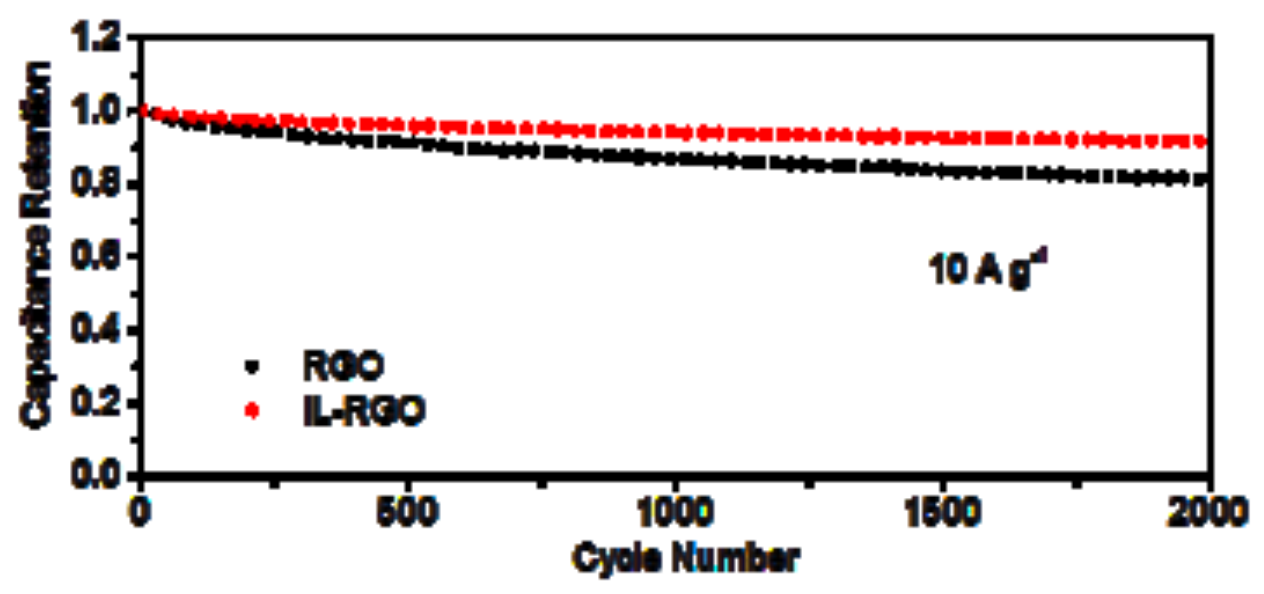


(a)

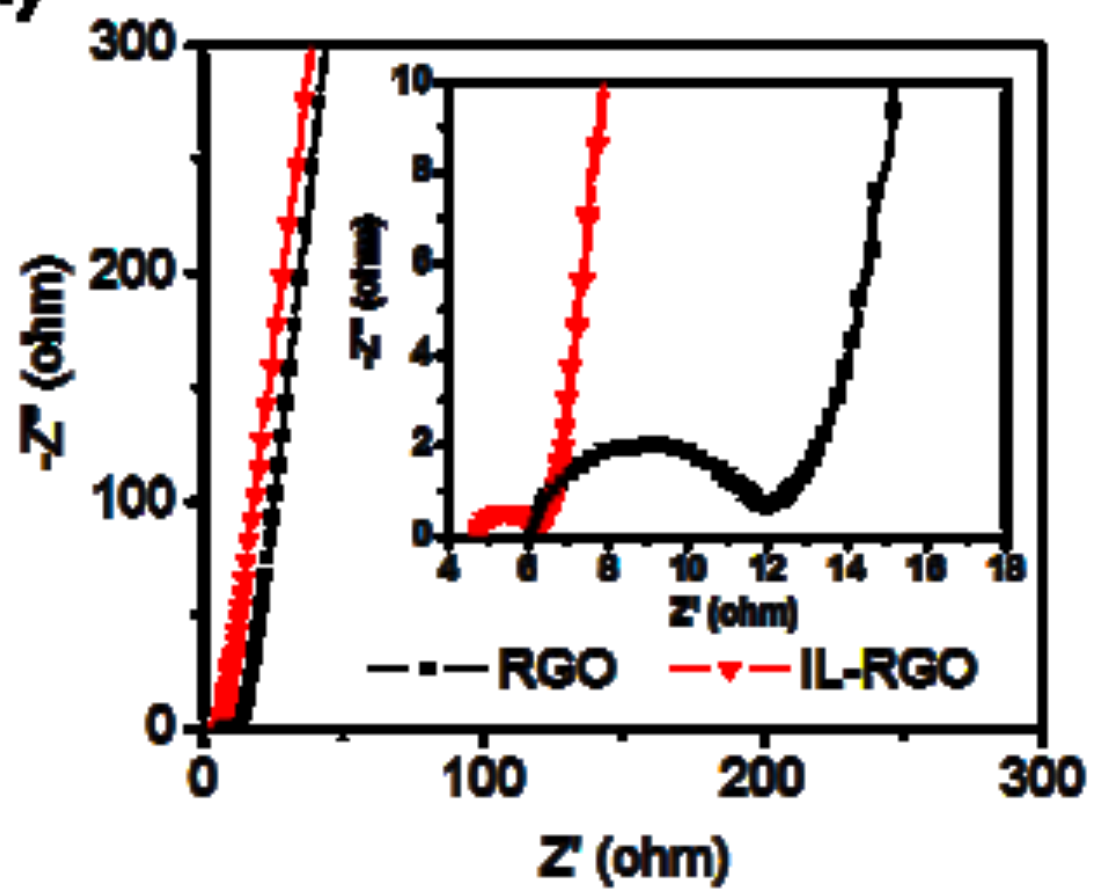

(b)

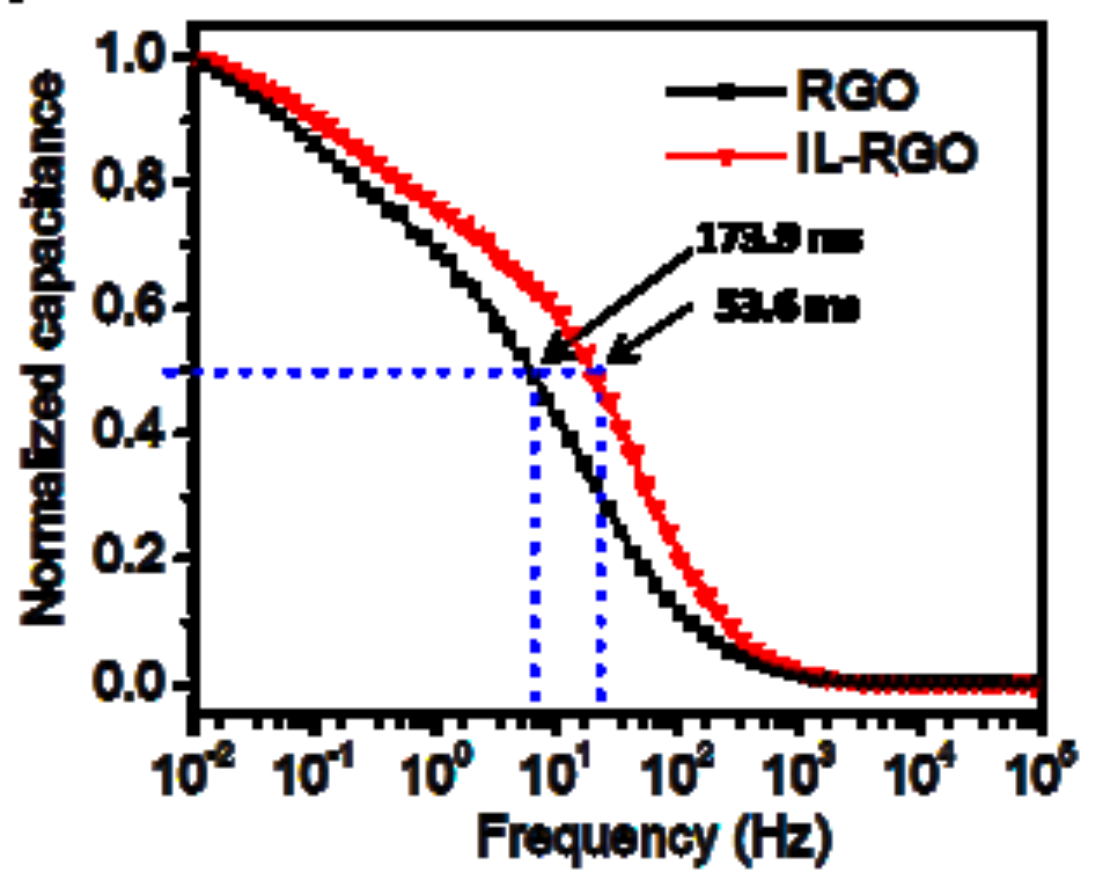


Graphical abstract
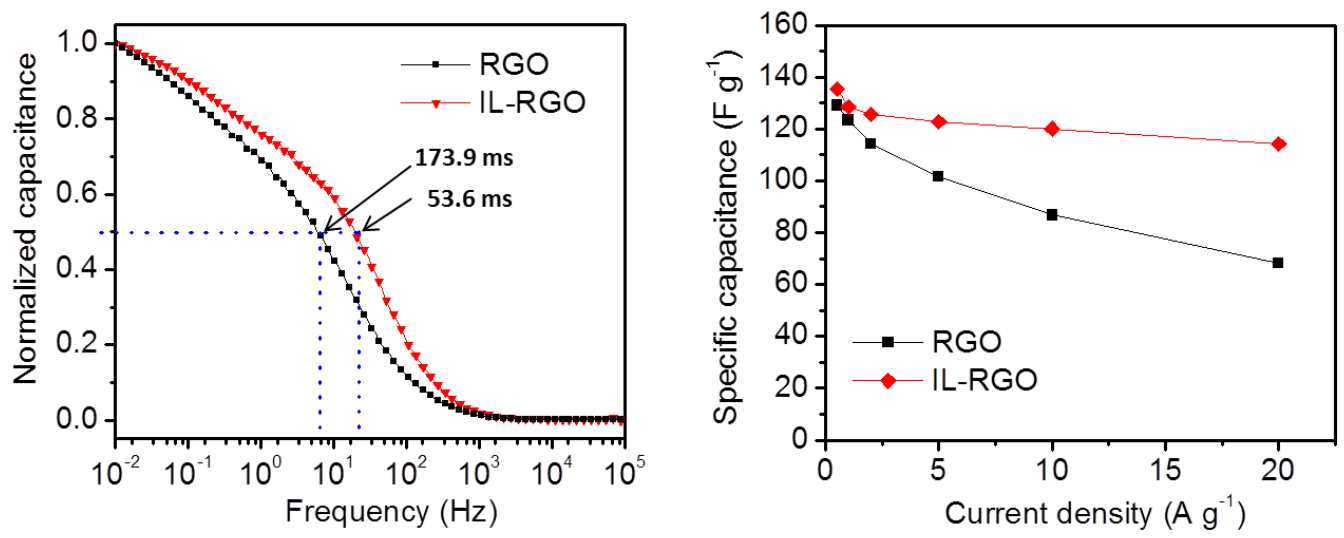

\section{Textual abstract :}

Ionic liquid modified reduced graphene oxide exhibits improved rate capability for supercapacitors using the same ionic liquid electrolyte. It is attributed to improved compatibility between the modified electrode and the same ionic liquid electrolyte. 